\title{
Base Station System Configurations for Future Universal Mobile Telecommunication Systems
}

\author{
Jos A. M. Nijhof, Member, IEEE, Irvan S. Dewantara, Ad J. M. Roovers, \\ and Ramjee Prasad, Senior Member, IEEE
}

\begin{abstract}
This paper presents a study of base station system configurations for the Universal Mobile Telecommunication System (UMTS). The base station system (BSS), being the interface between the mobile terminal and fixed network, should be capable of handling a huge amount of traffic, mobility, and a high signaling load which is expected in the UMTS. Several functions that should be supported by BSS are envisaged. Special attention is given to the mobility functions (e.g., handover). Functions that are needed to perform a handover are identified. This results in a generic handover functional model. Possible allocation scenarios are examined, taking into account the interconnection methods for the BSS and considering the efficiency and processing delay. The possible scenarios are further evaluated for given traffic and mobility models in different areas and environments.
\end{abstract}

\section{INTRODUCTION}

$\mathrm{T}$ HE first generation analog mobile systems (e.g., NMT, AMPS, TACS, etc.) were introduced in 1980 and penetrated up to $10 \%$, while the second generation digital systems (e.g., GSM, ADC, JDC, etc.) were introduced in several countries in 1992 and are targeted to penetrate up to $30 \%$ of all calls. Looking at the fast increase in the mobile communications services demand, there is a general trend worldwide to develop third generation future personal communications systems (PCS) which are expected to be introduced by the end of 20th century and penetrate up to $50 \%$ of all calls. The European community is also supporting the development of a third generation PCS system called Universal Mobile Telecommunication System (UMTS). A consortium of 26 European institutions has started the development of this third generation system within the European RACE program (Research and Development in Advanced Communications technologies in Europe). UMTS intends: 1) to provide a wide range of mobile services comparable to those offered in the contemporary fixed network including high bit rate services; 2 ) to be a standardized system, supporting mo-

Manuscript received September 30, 1993. This is an expanded version of a paper originally presented at the 43rd IEEE Vehicular Technology Conference.

J. A. M. Nijhof and R. Prasad are with the Telecommunications and Traffic-Control Systems Group, Delft University of Technology, 2100 GA Delft, The Netherlands.

I. S. Dewantara is with the Agency for the Assessment and Application of Technology (BPPT), Indonesia.

A. J. M. Roovers is with Royal PTT Nederland NV, PTT Research, 2260 AK Leidschendam, The Netherlands

IEEE Log Number 9403203.

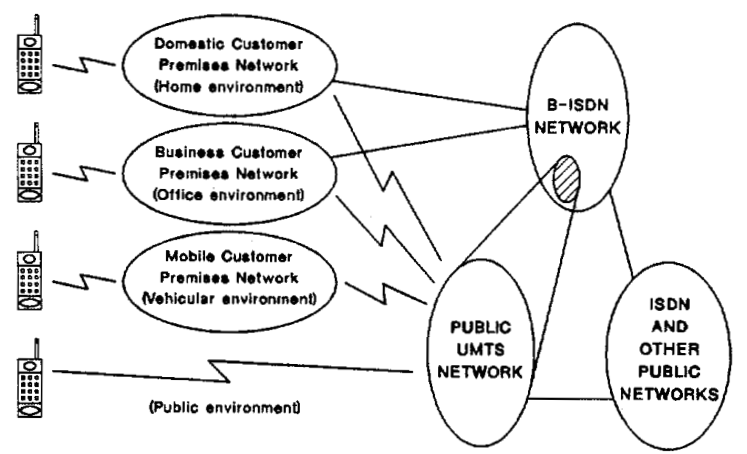

Fig. 1. Environment integration for UMTS subnetworks and fixed networks.

bile access in almost any environment, e.g., indoors, outdoors, private, and public; and 3 ) to be used by a large number of subscribers, using a range of terminals from the basic pocket telephone to more sophisticated terminals. Using the pocket telephone, communications services will be available on almost any location, indoor or outdoor, city or rural area, in home, office, or street. The availability of cheap pocket telephones will lead to a mass market where a large number of users will apply UMTS for their voice communication. This fact, coupled with the additional high bit rate services, means that UMTS must support very high traffic densities. Fig. 1 shows the integration of environments for UMTS networks and fixed networks.

Four environments associated with these subnetworks are: 1) home environment (Domestic Customer Premises Network, DCPN), 2) office environment (Business Customer Premises Network, BCPN), 3) vehicle environment (Mobile Customer Premises Network, MCPN), and 4) public environment. A more detailed introduction of UMTS and its objectives is discussed in [1]-[6]. Fig. 2 shows the UMTS network architecture and the role of the base station systems (BSS) within this architecture. In this paper, first, the mobility functions to be supported by the BSS are listed. Special attention will be given to the handover function since it will have the most significant impact on the BSS. The required network elements in the BSS have been determined, as well as possible interconnection topologies. In the next step, we have evaluated the allocation of the functions onto the network elements. 


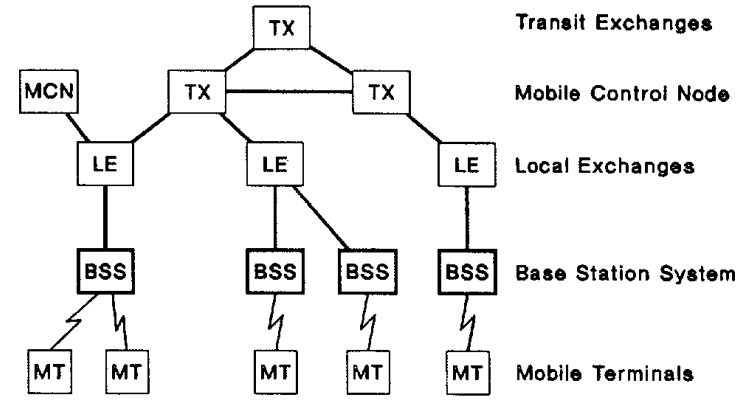

Fig. 2. UMTS network architecture.

Some allocation scenarios are proposed and analyzed with respect to the processing delay and the load on interfaces. Traffic and mobility characteristics for several environments obtained from previous studies [7], [8] are discussed. These data are used to evaluate the average processing delay and the load on the interfaces. Finally, recommendations for BSS network topologies in representative environments are given.

\section{Base Station System Functionalities}

Four types of services have been defined for the UMTS, namely, conversational, distribution, collection, and messaging services [2]. Five function groups providing these services have been determined: call and connection, mobility, charging, operation and maintenance (O\&M), and transport and conversion [5]. Some of these functions need support of the BSS. This section, however, focuses only on mobility functions by describing location updating, paging, and handover.

Location updating is a function by which the network keeps track of the location of the user. In case the mobile terminal leaves its location area, location updating takes place between the mobile entity and the network. From a previous study [6], it was concluded that the location area will be larger than the cell site area since it reduces the number of location updates that have to take place. The BSS only has to transfer the signaling information. The control of the location updating procedure is not likely to be allocated in the BSS. To enable a mobile entity to identify its location, a BSS should periodically transmit the area identifies to the terminal over the radio interface. The objective of the paging procedure is to find the exact position of the called party within the location area. On receipt of the broadcast "paging request"' from the network, the mobile terminal of the called party responds by sending a "paging response." Like the location updating procedure, the paging control entity can be located on the MCN (Mobile Control Node). The BSS is instructed to perform the paging and to receive the "paging response" from the terminal.

Handover is the change of the channel and/or the point of attachment during a call. The purpose of the handover procedure is to ensure the continuity of a call. Handover can occur for several reasons, e.g., radio propagation

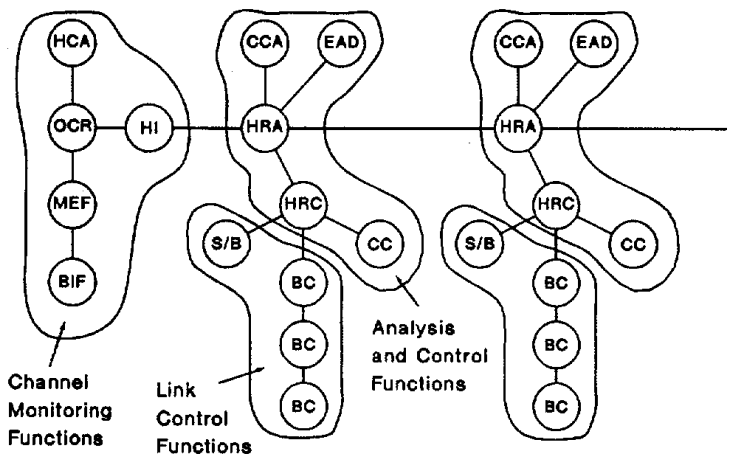

Fig. 3. Generic handover functional model.

characteristics, traffic distribution, O\&M activities, or movement of the user. The number of handovers taking place in a UMTS micro- and picocellular network will be substantially larger than in pure macrocellular networks. In order to avoid extensive signaling, the handover control should be performed on a low hierarchical level in the network. Therefore, some of these functions will be allocated in the BSS. Since handover will put the strongest requirements on the design of the BSS, only the handover functionality will be analyzed in more detail. This analysis will be used to evaluate several BSS options. In the handover process, two phases can be distinguished. 1) The monitoring phase: In this phase, the quality of the current and possible candidate radio links are measured periodically. The results are compared with predefined threshold values. A handover is initiated when necessary. 2) The handover handling phase: In this phase, a new point of attachment is determined, new links are set up, and old links are released. Possibly, also, a rerouting procedure is initiated.

According to these phases, a Generic Handover Functional Model as presented in Fig. 3 has been derived. The Broadcast Information Function (BIF), Measurement Function (MEF), Handover Criteria Analysis (HCA), and Old/Candidate Recognition (ORC) are performing the monitoring phase. A handover request will be issued to the Handover Initiator (HI) when necessary. The handover request will be analyzed by the Handover and Recognition Analysis function (HRA). The HRA is supported by the Call Continuity Analysis function (CCA) which analyzes the call and handover parameters (e.g., break time) and by the (Efficient) ANO Determination (EAD) function which determines where the bridge/switch between the new and the old link should be located (ANO $=$ Attach New to Old). If an HRA cannot handle the handover request, its task is passed to the next HRA. The HRA also determines which entity controls the handover. The Handover and Rerouting Control (HRC) supervises the handover process. It requests for new links from the ANO point to the mobile user through the new base station. The link setup will be performed by the Bearer Control function (BC). Switching or Bridge (S/B) functions are used in the fixed network ANO point and in the mobile 
terminal. If required, the transfer of control parameters will be performed by the Call Continuity (CC) function. After the mobile terminal has been instructed to change its channel, the old links will be released.

\section{Base Station System Architecture}

Since a large number of BSS's is needed, we should try to reduce their costs by using all resources and functions in the BSS as efficiently as possible. In this section, we will evaluate possible network entities and their interconnection schemes, as well as possible handover function allocation scenarios.

1) BSS Network Entities: It is clear that at least a transceiver function is needed to cover a cell. All functions related to the radio control will be collocated with the transceiver function. The network entity containing at least these functions will be further called the Base Transceiver Station (BTS). Each cell site has at least one BTS. It has been mentioned before that some mobility functions should be performed in the BSS. Allocating all mobility functions in the BTS will increase the cost, size, and complexity of the BTS. Therefore, a Base Station Controller (BSC) is defined which controls the BTS. The BSC will include some functions commonly used by several BTS's. A BSC controls one or more BTS's.

2) Interconnection Topologies: Several topologies can be used to interconnect the BSS entities: mesh, tree, ring, and bus topology. A full mesh topology for a BSS is not economically feasible since only adjacent entities interact directly. A partial mesh topology is probably more suitable. A tree topology is an option especially when the control is centralized. A bus or ring topology employs a fully shared broadcast transmission facility which can be used to interconnect BSC's. In this paper, we will only consider two options for the evaluation of the interconnection scenarios: 1) a direct connection between signaling entities (e.g., BSC-BSC); and 2) signaling through a higher hierarchical level (e.g., BSC-LE-BSC).

3) Allocation Scenarios of Handover Functions: Several scenarios can be proposed to map the generic handover functional model onto the network entities. We have distinguished three groups of functions in the generic functional model: the channel quality monitoring functions, the analysis/control functions, and the link control functions Since monitoring the channel quality is closely related to the radio interface, the MT and BTS are considered to perform all related functions. The MCN and BSC are considered to perform the handover control functions. Such functions will not be allocated in the LE and TX since they do not support mobility functions. As mentioned before, the BTS should be kept as simple as possible. Therefore, there will be no control functions allocated in the BTS. Except for the MCN, all network entities are assumed capable of setting up bearer links and constructing a bridge or switch. We have evaluated a number of function allocation and interconnection schemes. The performance of the handover procedure has been defined
TABLE I

INTRA-BSC HANDOVER

\begin{tabular}{|c|c|c|c|c|}
\hline interconnection & handover control & switching point & delay (a.u.) & Hlinks \\
\hline star & BSC & BSC & 48 & 7 \\
\hline BTS-BTS & BSC & BTS & 48 & 13 \\
\hline star & MCN & BSC & 48 & 23 \\
\hline
\end{tabular}

TABLE II

INTRA-LE HANDOVER

\begin{tabular}{|c|c|c|c|c|}
\hline interconnection & handover control & switching point & delay (a.u.) & \#links \\
\hline \hline star & BSC & LE & 60 & 15 \\
\hline BSC-BSC & BSC & BSC & 60 & 11 \\
\hline BTS-BTS & BSC & BTS & 58 & 13 \\
\hline star & MCN & LE & 80 & 21 \\
\hline BSC-BSC & MCN & BSC & 80 & 25 \\
\hline BTS-BTS & MCN & BTS & 78 & 27 \\
\hline
\end{tabular}

by two criteria: 1) the processing delay due to the execution of the functional entities, and 2) the number of physical links involved. The delays, in arbitrary units (a.u.), have been estimated by comparing the complexities of the functional entities. It is assumed that they are independent of the allocation to physical entities. A number of options have been investigated. These options concern the allocation of the handover control function (BSC or $\mathrm{MCN}$ ) and the interconnection of the BSS entities. The location of the switching point follows directly from the previous options. The performance of the handover procedure for various handover cases and options has been obtained, and the most important results are summarized in Tables I-III.

Table I shows that the processing delays are equal for all three scenarios. However, the number of external signaling links for the first scenario is smaller than for the other two. It can be concluded that the first scenario is the most efficient one for intra-BSC handover.

Table II shows some scenarios of intra-LE handover. Both the processing delay and the number of links will be smaller if the handover control functionality is allocated in the BSC instead of in the MCN. Direct linking of the BSC's will further minimize the load of the interfaces. The same conclusion could be drawn with respect to the allocation of the handover control function in the interLE handover cases, as depicted in Table III. However, it would imply that the BSS should be able to control LE's (using Signaling System No. 7). For security reasonsone of the reasons for separate network and access signaling-inter-LE handover can only be controlled from the fixed network (MCN). The performances and reasoning of the inter-MCN handover cases (not shown) are similar to the ones of inter-LE handover.

From the results, we can conclude that it is preferred to allocate the control functions in the BSC's for intra-LE handover cases since it will minimize the processing delay and the load on the interfaces. However, some issues concerning the capacity of the BSC, the handover and BSS requirements with respect to the handover processing de- 
TABLE III

INTER-LE HANDOVER

\begin{tabular}{|c|c|c||c|c||}
\hline interconnection & handover control & switching point & delay (0.u.) & \#links \\
\hline star & BSC & LE & 64 & 19 \\
\hline LE-LE & BSC & LE & 62 & 17 \\
\hline BSC-BSC & BSC & BSC & 60 & 11 \\
\hline BTS-BTS & BSC & BTS & 58 & 13 \\
\hline star & MCN & LE & 84 & 25 \\
\hline LE-LE & MCN & LE & 82 & 23 \\
\hline BSC-BSC & MCN & BSC-BSC & 80 & 25 \\
\hline BTS-BTS & MCN & BTS-BTS & 78 & 27 \\
\hline
\end{tabular}

lay, and the load of interfaces also have to be taken into consideration. They have to do with the traffic, mobility, and environment characteristics. In an environment where the radio power drops rapidly, the constraints on the delay will be more important. In an area with much traffic and a huge number of mobile subscribers, the amount of signaling and the load on the interfaces will be a crucial issue.

\section{Traffic and Mobility Characteristics}

Traffic and mobility characteristics have a major impact on the signaling load and on the mobility-related events (e.g., location updating, handover, call attempt, etc.). Since the UMTS will allow a large amount of services and users in the future, a huge amount of traffic will load the network far more than is the case with the current systems. A study of these aspects will give an important input for designing the UMTS, especially in designing the BSS.

Amsterdam, The Netherlands, is taken as an example city for our study. The example city is represented by concentric rings, labeled urban center, urban, suburban, rural, and remote rural as illustrated in Fig. 4. Each ring is further divided into a number of environments: busy spots (in the center of a metropolitan area), business indoor environments in a high-density office area, domestic indoor environments, motorways, and public outdoors. The present study is based on [7] and [8].

The city center area, i.e., urban center, is a circle with a $1 \mathrm{~km}$ radius and consists primarily of busy spots, office buildings, home environments, and a very small segment of motorway environments and others. Call and subscriber characteristics for this city center area are shown in Table IV. The terminal subscribers are divided into three types, namely, car (terminal mounted in a car), pedestrian (handheld telephone), and seated subscribers (fixed terminal in building). In Table IV, SDF, Vped, and Vcar represent the service data function, the average speed of a pedestrian, and the average speed of a car, respectively.

The number of call attempts, the handover rate, the location updating, and the paging rate are given in Table $\mathrm{V}$.

Assume that the UMTS will allow a base station in home environments, integrated to the UMTS network, and will allocate bandwidth to picocells. Then it is possible to perform a handover to a public outdoor base station when

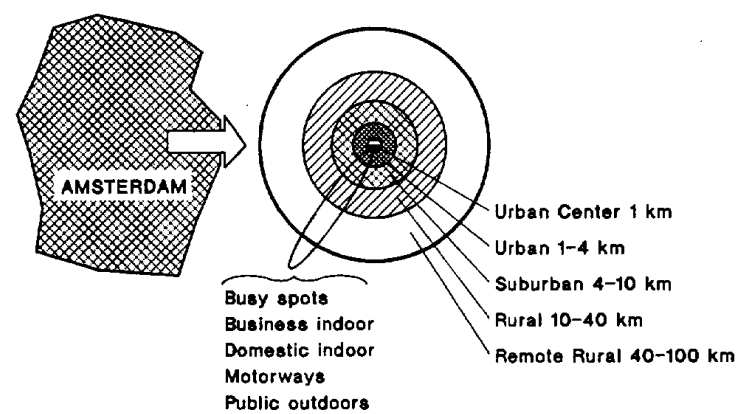

Fig. 4. Concentric model of a town.

TABLE IV

Cell and Subscriber Characteristics for the City Center Area

\begin{tabular}{|c|c|c|c|c|c|}
\hline & Busy Spot & Business & Domestic & Motorway & Other \\
\hline $\begin{array}{l}\text { Topology: } \\
\text { Number of subscribers } \\
\text { Cell Type } \\
\text { Cell radius (km) } \\
\text { SDF(car:pedestrian: } \\
\text { seat) } \\
\text { Mobility: } \\
\text { Vcar (km/hour) } \\
\text { Vped (km/hour) }\end{array}$ & $\begin{array}{c}30,000 \\
\text { micro } \\
0.09 \\
3: 4: 3 \\
\\
20 \\
5\end{array}$ & $\begin{array}{c}31,500 \\
\text { pico } \\
0.015 \\
0228\end{array}$ & $\begin{array}{c}1,300 \\
\text { pico } \\
0.10 \\
0: 2: 8\end{array}$ & $\begin{array}{c}125 \\
\text { macro } \\
1 \\
1: 0: 0\end{array}$ & $\begin{array}{c}47 \\
\text { macro } \\
1 \\
3: 4: 3\end{array}$ \\
\hline
\end{tabular}

TABLE V

Signaling Event Characteristics in the City Center Area

\begin{tabular}{|ll|c|c|c||}
\hline & & Busy Micro & Business Pico & Macro \\
\hline CALL ATTEMPT: (hour) & & & & \\
Call attempt rate & & 130,000 & 4,200 & 7,000 \\
HANDOVER: (hour) & & & & \\
Inter cell handoverhour: & Car & 1,900 & & 560 \\
Inter Area handover/hour: & Pedestrian & 320 & 180 & 20 \\
& Car & 14,000 & - & 560 \\
Intra Area handover/hour: & Pedestrian & 2,300 & 250 & 20 \\
& Car & 140,000 & - & - \\
& Pedestrian & 23,000 & 750 & - \\
LOCATION UPDATING: & & & & \\
Location updating rate/hour: & Car & 58,000 & & 2,100 \\
& Pedestrian & 19,000 & 1,700 & 330 \\
PAGING: (fhour) & & & & \\
Paging rate & & 48,000 & 1,300 & 6,600 \\
\hline
\end{tabular}

a subscriber is leaving the house. The number of subscribers moving out a home environment is rather difficult to estimate. But since the mobility of the subscribers in this environment is very low, the number of handovers, location updates, etc., is also small.

The busy environment will use bandwidth allocated to microcells. Since $64 \%$ of the city center is a busy spot environment [7], 80 cells with a radius of $90 \mathrm{~m}$ should be implemented. Further, an overlay macrocell is employed, not only to serve the subscribers on motorways and in other environments, but also to be used in case the radio capacity in microcells becomes insufficient to serve the traffic demand. Table $\mathrm{V}$ shows the number of traffic and mobility procedures per hour. Two types of handover are shown in Table V, i.e., intraarea and interarea handover. Intraarea handover rate reflects the number of calling subscriber moving from one cell to another within the city center area, while interarea handover is dealing with calling subscribers moving out of the city center area. The 
TABLE VI

Cell and Subscriber Characteristics for One Circled Urban ARea (RADIUS $1.5 \mathrm{~km}$ )

\begin{tabular}{|l|c|c|c|c|c|}
\hline & Busy Spot & Business & Domestic & Motorway & Other \\
\hline Topologv: & & & & & \\
Number of subscribers & 2,700 & 39,000 & 21,000 & 850 & 550 \\
Celll Type & micro & pico & pico & macro & macro \\
Cell radius (km) & 0.2 & 0.015 & 0.1 & 1.5 & 1.5 \\
SDF (car:pedestrian:scat) & $3: 4: 3$ & 0.28 & $0: 2: 8$ & $1: 0.0$ & $3: 4: 3$ \\
Mobility: & 30 & - & & & 50 \\
Vcar (km/hour) & 5 & 3 & 3 & - & 5 \\
Vpod (km/hour) & & & & \\
\hline
\end{tabular}

total intraarea handover rate in busy spot microcells is approximately $160000 / \mathrm{h}$. Such a number of events should not be handled by a single concentrated MCN (Mobile Control Node), but it would be better if it would be distributed over a number of control points.

The urban area is defined between $1-4 \mathrm{~km}$ from the city center. Assume that the urban area shown in Fig. 4 can be divided into a number of circled areas with a radius of $1.5 \mathrm{~km}$. The number of subscribers in one circled area for busy spot, business, domestic, motorway, and other areas are $2700,39000,21000,850$, and 550, respectively. The number of cars, pedestrians, and seated terminals is 1800,5500 , and 17800 , respectively. Some characteristics for one circled urban area are presented in Table VI.

$50 \%$ of the urban is domestic, $11 \%$ business, and $34 \%$ public. For office environments, a similar cell layout as in the city center area is applied. Three microcells will cover the busy spot environment. A macrocell of radius $1.5 \mathrm{~km}$ could cover the motorway and other environments. To cover the complete urban area, seven circled areas as defined above are needed. The signaling event characteristics for the business environment are similar to the ones in Table V. Table VII presents the signaling event characteristics for micro- and macro-cells. From Table VIII, we see that the number of signaling events caused by mobility is much lower than in the city center area.

Employing a dedicated macrocell for car subscribers will further reduce the signaling events. The suburban area is located between $4-10 \mathrm{~km}$ from the city center. The area is mainly characterized by domestic and public outdoor environments. We have divided the suburban area into a circled area with a radius of $3 \mathrm{~km}$. The number of subscribers in the business, busy spot, domestic, motorway, and other areas is $1000,71000,25500,1600$, and 2400 , respectively. Table VIII shows the cell and subscriber characteristics for the suburban area.

A similar scenario as in the city center area is used for the office environments. For busy spots, three cells are needed, and, similarly, for the motorway environment, we assume that the overlay macrocell will cover this area. Thus, we have an overlay macrocell which covers the whole area under study, three microcells covering busy spots, and a number of cells employed in the business and domestic indoor environments.

Table IX gives the results of the call attempt rate, handover rate, location updating rate, and paging rate in this area. Ten circle areas identical to the circle area studied
TABLE VII

Signaling Event Characteristics in an Urban AREa

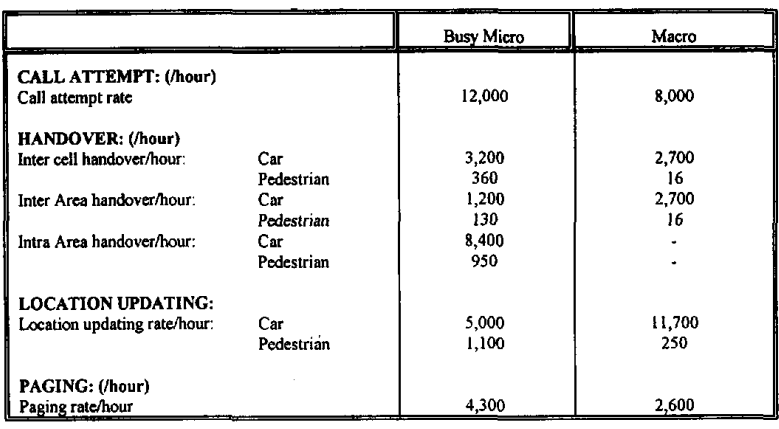

TABLE VIII

Cell and Subscriber Characteristics for One Circled Suburban AREA

\begin{tabular}{|l|c|c|c|c|c|}
\hline & Busy Spot & Business & Domestic & Motonvay & Other \\
\hline Topology: & & & & & \\
Number of subscribers & 1,000 & 71,000 & 25,500 & 1,600 & 2,400 \\
Ceell Type & micro & pico & pico & macro & macro \\
Cetll radius (km) & 0.17 & 0.15 & 0.1 & 3 & 3 \\
SDF (car:pedestrian:seat) & $3: 4: 3$ & $0: 2: 8$ & $0: 2: 8$ & $1: 0.0$ & $3: 4: 3$ \\
& & & & & \\
Mobility: & & & & 100 & 70 \\
Ycar (kmhour) & 40 & 3 & 3 & - & 5 \\
Vped (km/hour) & 5 & & & \\
\hline
\end{tabular}

TABLE IX

Signaling Event Characteristics in the Suburban ARea

\begin{tabular}{|c|c|c|c|c|c|}
\hline & & Busy Micro & Domest. Micro & Macro & Total \\
\hline $\begin{array}{l}\text { CALL ATTEMPT: (hour) } \\
\text { Call attempt rate }\end{array}$ & & 4,400 & 62,500 & 18,000 & 84,900 \\
\hline $\begin{array}{l}\text { HANDOVER: (hour) } \\
\text { Inter cell handover/hour: }\end{array}$ & $\begin{array}{l}\text { Car } \\
\text { Pedestrian }\end{array}$ & $\begin{array}{c}1,800 \\
150\end{array}$ & 20 & $\begin{array}{c}23,500 \\
70\end{array}$ & - \\
\hline Inter Area handover/towr: & $\begin{array}{l}\text { Car } \\
\text { Pedestrian }\end{array}$ & $\begin{array}{c}30 \\
3\end{array}$ & - & $\begin{array}{c}2,700 \\
30\end{array}$ & $\begin{array}{c}2,730 \\
120\end{array}$ \\
\hline Intra Area handoverhour: & $\begin{array}{l}\text { Car } \\
\text { Pedestrian }\end{array}$ & $\begin{array}{c}5,250 \\
450\end{array}$ & 1,230 & - & $\begin{array}{l}5,250 \\
1,680\end{array}$ \\
\hline $\begin{array}{l}\text { LOCATION UPDATING: } \\
\text { Location updating rate: }\end{array}$ & $\begin{array}{l}\text { Car } \\
\text { Pedestrian. }\end{array}$ & $\begin{array}{l}130 \\
23\end{array}$ & $\overline{1,600}$ & $\begin{array}{c}22,300 \\
500\end{array}$ & $\begin{array}{c}22,430 \\
2,123\end{array}$ \\
\hline $\begin{array}{l}\text { PAGING: (hour) } \\
\text { Paging rate }\end{array}$ & & 1,600 & 19,400 & 6,100 & 27,100 \\
\hline
\end{tabular}

above are needed to cover the suburban area. In the rural area, the public outdoor environment covers $90 \%$ of the whole area. A number of macrocells with a radius of 7.7 $\mathrm{km}$ should be employed. The number of subscribers in this area is 2800 (domestic), 1400 (motorway), and 5000 (other). The crossing rate/hour/cell is 24000 . The number of inter cell handovers is $3300 / \mathrm{h}$, of location updating $12000 / \mathrm{h}$, and of call attempt 30000 . The paging rate is $9900 / \mathrm{h}$.

The remote area has a very low population density consisting mainly of public outdoor areas and a very small area of motorway and domestic environments. Macrocells with a radius larger than $25 \mathrm{~km}$ are used to cover the whole area. 


\section{Conclusions}

Base station system functionalities, its architecture, traffic, and mobility characteristics for the third generation UMTS system have been investigated in this paper. A handover generic functional model is developed based upon the assumption that a set of functions is common to the variety of services. The definition of such a set of generic functions would limit the complexity of the network equipment.

Using intra-BSC, intra-LE, and inter-LE handover (Tables I, II, and III), it can be concluded that the delay and load performances will be enhanced if the handover control function is allocated in the BSC's. Direct interconnection links between control nodes (BSC-BSC) will further improve the performance. However, these solutions will increase the complexity and costs of the network. Therefore, this will only be implemented in an area where it is really needed. The area with a large amount of traffic and high mobility will benefit from this solution. In microcell areas which are surrounded by buildings, the signal power will decrease rapidly. In such areas, the handover handling should be fast. Traffic and mobility characteristics have been studied for a typical European city in The Netherlands-Amsterdam. It has been observed that the number of mobility events occurring in a city center area is remarkably larger than in business, domestic, or other areas. Therefore, the signaling loads and processing delays at the interfaces will also be more stringent. More attention should be paid to this area.

The amount of traffic in a business environment is, as expected, large. However, the handover rate in this environment is low. Therefore, it is recommended to centralize the handover control in one BSC. In domestic environments with private subnetworks, the mobility of the subscribers leaving the home is difficult to predict. The "mobility model" cannot be used here. However, the frequency of people leaving or coming in their house while having a call is low.

A possible solution to minimize the handover rate is to use a separate base station for highly mobile subscribers. Using this scenario, the amount of mobility-related signaling can be significantly reduced, especially in a city center area where a huge number of subscribers should be served. However, this requires more bandwidth for the macrocells than initially determined. The calculation results presented in this paper can be used to estimate the traffic and signaling load on the BSS network. In the case where the number of events in a particular area is extremely high, three solutions are proposed. First, use larger cells to reduce the number of mobility procedures. Second, direct connections between base stations on the fixed network layer will reduce the load on the BSS interfaces. The third solution is to distribute the control functions in a lower level of the network to improve the network performance.
ACKNOWLEDGMENT

The authors are thankful to $W$. van Schagen for preparing the manuscript.

\section{REFERENCES}

[1] T. Parrot, "RACE mobile universal personal communications,"' Mo bile Telecommun. News, p. 26.B-Allocation scenario of handover functions, Nov. 1990.

[2] "Systems description-Part 1, Universal mobile telecommunications system," CEC Deliverable, 43/PRL/SG1/DS/A/100/a2, June 10 , 1992.

[3] S. Chia, "The univesal mobile telecommunications system," IEEE Commun. Mag., pp. 54-62, Dec. 1992.

[4] H. de Boer, M. Meijer, and E. Buitenwerf, "Network aspects for the third generation mobiles,"' in Proc. GLOBECOM'91, Phoenix, AZ, Dec. 1991, pp. 1517-1522.

[5] "Final report on fixed network aspect in UMTS," CEC Deliverable, 43/RNL/FN12/DS/A/067/b1, Dec. 13, 1991.

[6] "Location areas, paging areas and the network architecture," $C E C$ Deliverable, R2066/PTTNL/MF1/DS/P/001/b1, May 1, 1992.

[7] B. J. M. Stortelder and H. E. Voortman, "Traffic models and statistics," CEC Deliverable, 43/RNL/MS4/DS/A/028/b1, Jan. 18, 1990.

[8] "System framework, CEC Deliverable, 43/PRL/SG1/DS/B/105/b1.

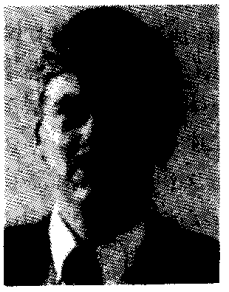

Jos A. M. Nijhof (M'93) was born in Rijswijk, The Netherlands, in 1945 . He received the M.Sc degree in electrical engineering from the Delft University of Technology in 1971.

He then joined the TU Delft Centre for Traffic and Transportation Engineering as a Research Coordinator. Since 1975 he has been with the Telecommunications and Traffic Control Systems Group of the Delft University of Technology, where he is now an Associate Professor of Data Communications Systems and Networks. He contributes regularly to courses and seminars on data communications, network architectures, mobile communications, and performance analysis of telecommunication systems. His research interests are protocols and access techniques for broad-band and wireless communications networks.

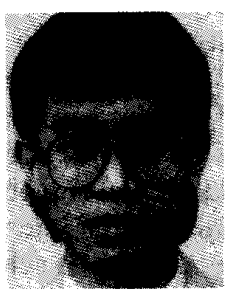

Irvan S. Dewantara was born in Surakarta, In donesia, on May 2, 1965. He received the Master's degree in electrical engineering from Delft University of Technology in 1993.

While pursuing his degree, he was with PTT Research, The Netherlands, participating in the RACE Progam Mobile networks. He is currently working as a Research Engineer at the Agency for the Assessment and Application of Technology (BPPT), Indonesia. His research interests are in the field of network planning for mobile commu-

nications.

Ad J. M. Roovers was born in Breda, The Netherlands, on August 24, 1961. He received the M.Sc. degree in applied physics and the Ph.D. degree in technical sciences from the University of Twente, The Netherlands, in 1985 and 1989, respectively.

After his military service and a postdoctoral position, he joined PTT Research as a Research Engineer in 1992. He is currently active in several mobile communication projects. His interests are personal communications and third generation mobile communication systems. 


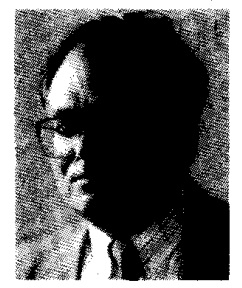

Ramjee Prasad (M'88-SM'90) was born in Babhnaur (Gaya), Bihar, India, on July 1, 1946. He received the B.Sc. (Eng.) degree from Bihar Institute of Technology, Sindri, India, and the M.Sc. (Eng.) and Ph.D. degrees from Birla Institute of Technology (BIT), Ranchi, India, in 1968 , 1970 , and 1979, respectively.

He joined BIT as a Senior Research Fellow in 1970 and became an Associate Professor in 1980. During 1983-1988, he was with the University of Dar es Salaam (UDSM), Tanzania, where he became a Professor of Telecommunications in the Department of Electrical Engineering in 1986. Since February 1988, he has been with the Telecommunications and Traffic Control Systems Group, Delft University of Technology, The Netherlands, where he is actively involved in the area of mobile, indoor, and personal radio communications. While he was with BIT, he supervised many research projects in the area of microwave and plasma engineering. At UDSM, he was responsible for the collaborative project "Satellite Communications for Rural Zones" with Eindhoven University of Technology, The Netherlands. He has published over 150 technical papers. His current research interest lies in packet communications, adaptive equalizers, spread-spectrum CDMA systems, and multimedia communications. He has served as a member of advisory and program committees of several IEEE international conferences. He has also presented tutorials on mobile and indoor radio communications at various universities, technical institutions, and IEEE conferences. He is also a member of a working group of European cooperation in the field of scientific and technical research (COST-231) project dealing with the "Evolution of land mobile radio (including personal) communications"' as an expert for The Netherlands.

Dr. Prasad is listed in the U.S. Who's Who in the World. He was Organizer and Interim Chairman of IEEE Vehicular Technology/Communications Society Joint Chapter, Benelux Section. He now is the elected Chairman of the joint chapter. He is also founder of the IEEE Symposium on Communications and Vehicular Technology (SCVT) in the Benelux, and he was the Symposium Chairman of SCVT'93. He is one of the Editors-in Chief of a new journal on wireless personal communications, and is also a member of the Editorial Board of other international journals, including IEEE Communications Magazine. He is the Technical Program Chairman of PIMRC'94 International Symposium to be held in The Hague, The Netherlands during September 1994, and also of the Third Communication Theory Mini-Conference in conjunction with GLOBECOM'94 to be held in San Francisco, CA, during November 1994. He is a Fellow of the IEE, a Fellow of the Institution of Electronics and Telecommunication Engineers, and a member of the New York Academy of Sciences. 